\title{
Analytical formulation of the integral square error for linear stable feedback control system
}

\begin{abstract}
The paper aims to introduce a method for the analytical formulation of the integral square error (ISE). In this manner, the aim of the research is to create a parametric solution of the ISE for linear continuous feedback control systems while the closed-loop system is stable and the difference between reference and output, or error, is strictly proper. The potential advantage of this technique is that it allows the finding of an analytical solution for the ISE criterion and hence it is well adopted when a parametric solution of the ISE for optimal control problems is needed. This method is also superior to the numerical methods, because it returns the exact solution of the ISE. Comparisons with a powerful numerical method are given to validate the proposed method.
\end{abstract}

Keyword: Performance index; Integral square error; ISE; Optimum control problem 